\title{
Ansätze zur Etablierung von Präzisionsmedizin bei der Parkinson- Krankheit mit dem Schwerpunkt Genetik
}

\section{Emerging concepts for precision medicine in Parkinson's disease with focus on genetics}

Autoren

Lara Stute $^{1,2}$, Rejko Krüger ${ }^{1,2,3}$

Institute

1 Parkinson Research Clinic, Centre Hospitalier de Luxembourg (CHL), Luxembourg, Luxembourg

2 Luxembourg Centre for Systems Biomedicine (LCSB), University of Luxembourg, Esch-Sur-Alzette, Luxembourg

3 Transversal Translational Medicine, Luxembourg Institute of Health (LIH), Strassen, Luxembourg

Schlüsselwörter

Präzisionsmedizin, Personalisierte Medizin, Stratifizierung, Parkinson-Krankheit, Genetik

Key words

Precision Medicine, Personalised Medicine, Stratification, Parkinson's disease, Genetics

eingereicht $\quad 30.01 .2020$

akzeptiert 28.03.2020

\section{Bibliografie}

DOI https://doi.org/10.1055/a-1149-2204

Fortschr Neurol Psychiatr 2020; 88: 558-566

(c) Georg Thieme Verlag KG Stuttgart · New York

ISSN 0720-4299

Korrespondenzadresse

Prof. Dr. Rejko Krüger

Director of Transversal Translational Medicine,

Luxembourg Institute of Health (LIH),

$1 \mathrm{~A}-\mathrm{B}$, rue Thomas Edison

L-1445 Strassen

Luxembourg

Tel.: +352 $26970-458$

Fax: +352 $26970-719$

E-Mail: rejko.krueger@lih.lu

\section{ZUSAMMENFASSUNG}

Während Parkinson mit seiner vielfältigen und sehr individuellen Kombination aus motorischen und nichtmotorischen Symptomen zunehmend genauer charakterisiert ist, nicht zuletzt durch die Untersuchung von großen
Patientenkohorten mit Deep-Phenotyping-Approach, folgt die Therapie weiterhin einem einheitlichen Schema. Durch bessere Stratifikation bieten PräzisionsmedizinAnsätze die Möglichkeit, die Behandlung und patientenzentrierte Versorgung zu verbessern. Spezifische Therapien für den Einsatz bei monogenetischen ParkinsonFormen, die aktuell untersucht werden, könnten helfen, Krankheitsmechanismen zu verstehen und dadurch auch zum Verständnis des idiopathischen Parkinson-Syndroms beitragen, sowie neue Behandlungsziele aufzeigen. Wir zeigen Daten zur Vorhersage von Wirksamkeit und Langzeit-Vorteil von aktuellen medikamentösen Behandlungen sowie von Tiefer Hirnstimulation (THS) im Kontext von wachsendem pharmakogenetischen Wissen. Konfrontiert mit asymptomatischen Trägern genetischer Mutationen (monogenetische Erkrankung) von variabler Penetranz und prodromalen Stadien wie REM-Schlaf-Verhaltensstörungen, zeichnen sich erste präventive Therapiestrategien ab. Ihr Einfluss auf die Krankheitsprogression und Aussichten für die klinische Praxis müssen adressiert werden.

\section{ABSTRACT}

The diverse and highly individual presentations of Parkinson's disease (PD) as a complex combination of motor and nonmotor symptoms are being increasingly well characterised not least through large patient cohorts applying deep phenotyping. However, in terms of treatment of PD, the approach is uniform and purely symptomatic. Better stratification strategies with better precision medicine approaches offer opportunities to improve symptomatic treatment, define first causative therapies and provide more patient-centred care. Insight from targeted therapies for monogenic forms of PD aiming at neuroprotection may pave the way for new mechanism-based interventions also for the more common idiopathic PD. Improved stratification of patients may support symptomatic treatments by predicting treatment efficacy and long-term benefit of current pharmacological or neuromodulatory therapies, e.g. in the context of emerging pharmacogenomic knowledge. Based on asymptomatic carriers with monogenic PD or patients with REM sleep behaviour disorder (RBD), first options for 
applying preventive treatments emerge. The implications of these treatment strategies in relation to disease progression, and the prospects of their implementation in clinical practice need to be addressed.

\section{Einleitung}

Während aktuell häufig über personalisierte Medizin gesprochen wird, ist damit meist das Konzept der Precision Medicine gemeint, das darauf abzielt, sensitive Parameter zu determinieren, um Patienten-Subgruppen zu definieren, die sich für bestimmte Therapien qualifizieren bzw. voraussichtlich besser auf diese ansprechen. Ziel dieser gezielten Therapie-Ansätze ist außerdem der Wunsch, Krankheitsverläufe zu verlangsamen und, wenn möglich, zu stoppen. Wie von Love-Koh und Kollegen treffend beschrieben, handelt es sich bei Precision Medicine um Ansätze, die der Patienten-Stratifizierung für eine bestimmte Therapie dienen - aufbauend auf spezifischen Charakteristika des Individuums [1]. Die dabei verwendeten Charakteristika sind variabel, gehen aber über rein demographische Faktoren hinaus und enthalten genetische Informationen, Verhaltensmerkmale, Umweltfaktoren und physiologische Charakteristika. In diesem Sinne können klinikogenetische Informationen als Einstieg gesehen werden, um hypothesenbasierte Therapien zu entwickeln. Zukünftig werden allerdings algorithmenbasierte Ansätze, basierend auf den Erkenntnissen großer Kohorten, in den Vordergrund treten. Sie definieren drei technologische Innovationen in der Präzisionsmedizin: (i) den Einsatz von komplexen Algorithmen auf großen (Patienten-/Gesundheits-)Datensätzen, (ii) die Nutzung von sensorbasierten Daten (z.B. durch digitale GesundheitsApps) sowie (iii) die Nutzung von individuellen biologischen Informationen durch „-omics“-basierte Daten zur Identifikation von Biomarkern.

Diese Ansätze haben sich u.a. in der Onkologie als erfolgreich erwiesen, wie z.B. im Falle der BRCA1- und BRCA2Mutation bei Brustkrebs- und werden im Rahmen von Prävention, Diagnose und Behandlung eingesetzt [2]. Sogenannte Basket Trials schließen Probanden - über verschiedene Tumorerkrankungen hinweg - aufgrund der molekulargenetischen Klassifikation zugrunde liegender Pathomechanismen ein [3].

Das Konzept der Präzisionsmedizin ist bislang für neurodegenerative Erkrankungen nicht etabliert, obwohl diese eine enorme sozioökonomische Herausforderung darstellen. Allein in Europa betrugen die Kosten für die Behandlung und Pflege der ca. 7 Millionen Demenz-Betroffenen 2008 ca. 160 Milliar-

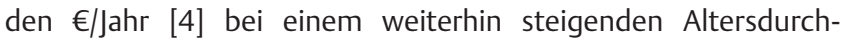
schnitt der Gesellschaft (Anteil der über 65-Jährigen in Europa aktuell bei $16 \%$, prognostisch bei $30 \%$ im Jahr 2030) [5]. Die Prävalenz der Parkinson-Krankheit in Deutschland lag 2016 zwischen 791 und 961/100000 mit einer totalen Patientenzahl in der Population der über 50-Jährigen 245912 und 296248 [6]. Für das Jahr 2040 wird mit einer Verdopplung der weltweit Erkrankten auf 12 Millionen von 6,1 Millionen Betroffenen ausgegangen [7].

\section{Stratifizierung}

\section{Aktuell gebräuchliche klinische Klassifikationen der Parkinson-Krankheit}

Wie andere neurodegenerative Erkrankungen weist auch die Parkinson-Krankheit eine große klinische Heterogenität auf. Die Patienten zeigen eine variable Kombination von motorischen und nichtmotorischen Symptomen mit sehr verschiedenen Krankheitsverläufen. Kurz: Jeder Patient hat seine eigene, individuelle Ausprägung der Parkinson-Krankheit und eine individuelle Trajektorie der Krankheitsprogression, dennoch sind die aktuellen Behandlungsmöglichkeiten immer noch sehr uniform.

Aktuell erfolgt eine Stratifizierung anhand der klinischen Zeichen, evaluiert mittels Anamnese, Untersuchung und systematischen Fragebögen, welche motorische Symptome, Anzeichen von atypischen Parkinson-Syndromen und nichtmotorische Symptome abdecken, auch bezüglich ihres Einflusses auf den Alltag.

Im klinischen Setting dominiert die Beschreibung der motorischen Subtypen als entweder Tremor-dominant, akinetischrigide, mit prädominanter Haltungs- und Gangstörung (Postural Instability Gait Disorder-PIGD ) oder gemischt, mit allerdings eingeschränkter Relevanz für die Einschätzung der Krankheitsprogression. Für den Tremor-dominanten Typ wurde vielfach ein milderer Verlauf gesehen oder eine distinkte Pathologie mit u.a. besser erhaltenen Neuronen der Substantia nigra angenommen [8]. Dagegen spricht, dass die Subtypen-Zuordnung in Krankheitsverlauf nicht statisch bleibt, sondern sich im individuellen Patienten ändern kann [8, 9].

Auch die Einordnung nach Erkrankungsalter ist leicht durchführbar und führt zu der Einteilung in eine Early-onset-Gruppe (EOPD) mit frühem Erkrankungsbeginn bis zum 40. Lebensjahr (von einigen Autoren bis zum 45./50. Lebensjahr erweitert [10, $11])$, die sich nochmals in eine Juvenile-onset-Gruppe (JOPD) bis zum 20. Lebensjahr und eine Young-onset-Gruppe (YOPD) bis zum 40. (respektive 45./50. Lebensjahr) aufteilt. Das Auftreten nach dem Alter von 40 Jahren wird demnach als Late-onset-Form definiert und als typisches Erkrankungsalter der idiopathischen Form der Erkrankung angesehen (steigende Inzidenz mit dem Alter). Studien, die Patienten in Bezug auf ihren Erkrankungsbeginn verglichen haben, beschreiben größtenteils einen milderen Verlauf bei EOPD-Patienten im Vergleich zu einem späteren Erkrankungsbeginn und seltener kognitive Defizite, allerdings mit einer erhöhten Rate von motorischen Komplikationen wie Dyskinesien [12]. Während die Inzidenz der Parkinson-Krankheit im höheren Alter steigt, ist der Einfluss auf Familienleben, Berufstätigkeit und die Einschränkung durch motorische Symptome bedeutender für den individuellen Patienten bei EOPD [12]. Strukturierte Klassifikationen des Krankheitsstadiums, wie die 1967 von Margarete Hoehn und Melvin Yahr vorgestellte Skala, 
können helfen, Meilensteine der Krankheitsprogression zu vergleichen [13]. Die Nutzung der Hoehn-und-Yahr-Skala ist weit verbreitet, jedoch fehlt laut Goetz [14] der lineare Zusammenhang, und es besteht eine Vermischung von motorischen Symptomen und Behinderung, wodurch ihr Nutzen für longitudinale Studien limitiert wird. Vor diesem Hintergrund wurde die Unified Parkinson's disease rating scale (UPDRS) entwickelt und durch die Movement Disorders Society (MDS-UPDRS) mit standardisierter motorischer Untersuchung und Fragebögen adaptiert, die auch nichtmotorische Symptome und Alltagsaktivitäten mit abdecken $[15,16]$.

Letztere trägt der zunehmenden Bedeutung von nichtmotorischen Symptomen (NMS) und ihrem Einfluss auf den Patienten Rechnung und führte zur Entwicklung von spezifischeren Fragebögen wie dem NMSQuest [17] oder spezifischen Fragebögen zu Teilaspekten wie Apathie [18]. Einige Autoren plädieren dabei für eine anatomo-klinische Korrelation, nach der NMS-Cluster erklärt werden können. Sie unterscheiden dabei drei Typen: ein Hirnstamm-Subtyp mit Schlafstörungen und autonomer Dysfunktion, ein limbischer Subtyp mit Depression, Fatigue und Gewichtsabnahme sowie ein kognitiver Subtyp (vor allem mit cholinerger Dysfunktion) mit Schwerpunkt auf kognitiver Beeinträchtigung, Apathie und Angststörungen [19]. Der Nachweis dieser Subtypen in einer klinischen, relativ heterogenen Kohorte in Italien war allerdings nicht möglich [20].

Für eine klinische Stratifizierung scheint eine Kombination von motorischen und nichtmotorischen Symptomen am sinnvollsten zu sein [21].

Um die klinische Diagnose der Parkinson-Krankheit zu bestätigen, ist nach wie vor eine neuropathologische Untersuchung unerlässlich, da es bislang keine validen Biomarker gibt, z.B. aus Körperflüssigkeiten oder -geweben, wie in der Bildgebung, die die Diagnose sichern bzw. eine Abgrenzung von atypischen Parkinson-Syndromen ermöglichen [22].

Wie können wir unser Wissen und unsere Vorhersagen verbessern und damit die Versorgung der individuellen Patienten?

\section{Neue Ansätze der Stratifizierung}

Den Limitierungen der einzelnen Klassifikationen kann mit der Nutzung von Algorithmen-basierter Stratifizierung und Integration von genetischen Informationen teilweise begegnet werden [23]. Ein Vorhandensein von genetischen Risikofaktoren kann in der Stratifizierung von Patienten als datenbasierte Grundlage dienen und mittels der Analyse von Gen-Umwelt-Interaktionen und der Untersuchung der beeinträchtigten Stoffwechselwege auch Hinweise für den jeweils zugrunde liegenden Pathomechanismus bei dem jeweiligen Parkinson-Patienten geben [24]. Eine Option ist dafür der Einsatz von Mendelian Randomization, wo genetische Varianten auf ihren kausalen Zusammenhang mit exogenen Risikofaktoren wie Kaffee- und Nikotinkonsum untersucht werden [25].

Um daraus allerdings neue kausale Therapien ableiten zu können, benötigen wir aufgrund der trotz allem großen klinischen Heterogenität und dem variablen klinischen Einfluss der relevanten Gene dafür große Kohorten mit detaillierter Phänotypisierung, die über einen großen Zeitraum verfolgt werden [26], und die Bereitschaft zum Daten- und Wissensaustausch zwischen verschiedenen klinischen Zentren.

\section{Genetische Stratifizierung als Konzept (monogenetisch bis GWAS)}

Parkinson ist eine größtenteils sporadische Erkrankung, deren größter Risikofaktor das Alter ist [27]. Zusätzlich spielen genetische sowie wahrscheinlich auch Umweltfaktoren eine Rolle [28].

Der genetische Einfluss kann als ein Kontinuum gesehen werden, bei dem an einem Ende des Spektrums die seltenen monogenetischen Formen hoher Penetranz („highly penetrant variants“) stehen, welche alleine verantwortlich für die Erkrankung sind [29]. Am anderen Ende stehen häufige genetische Varianten, die auch bei Gesunden beobachtet werden, jedoch mit einer unterschiedlichen Erhöhung des Lebenszeitrisikos, von der ParkinsonKrankheit betroffen zu sein, verbunden sind [29]. Allerdings gibt es auch bei den monogenetischen Parkinson-Formen eine variable Ausprägung in Bezug auf Krankheitsbeginn und Symptomausprägung bei identischen Genvarianten Betroffener der gleichen Familie, sodass die Beschreibung als monogenetisch als Vereinfachung angesehen werden kann [30].

Der Anteil der Patienten mit monogenetischen Formen aufgrund bekannter Mutationen wird auf bis zu 10\% geschätzt [31], kann aber in Abhängigkeit der Population selbst für eine spezifische Mutation, z.B. G2019 S-Mutation im LRRK2-Gen, bis zu 30\% betragen [32]. Der Anteil variiert abhängig von der betreffenden Population in Abhängigkeit vom Alter bei Erkrankungsbeginn, dem ethnischen Hintergrund und den getesteten Genen. Für den einzelnen Patienten erhöht sich dabei die Wahrscheinlichkeit durch junges Erkrankungsalter und positive Familienanamnese. Seltene, als kausal beschriebene Varianten sind aktuell in über 20 Genen bekannt, allerdings ist ihre Krankheitsrelevanz teilweise umstritten, da Studien zur Reproduzierbarkeit und funktionalen Validation nicht für alle sogenannten PARK-Loci vorliegen [30].

Genetische Screenings sind dank neuer Methoden kostengünstiger geworden, statt eines Whole-Genome-Sequencings oder Whole-Exome-Sequencings werden dabei häufig gezielt Veränderung einzelner Nukleotide angeschaut, als Hochdurchsatz-Genotypisierungs-Strategie. Zum Einsatz kommen dabei genetische Panels wie der NeuroChip, der seltene, pathogene sowie häufige Varianten abdeckt, die eine Rolle bei häufigen neurodegenerativen Erkrankungen spielen [33]. Zu den seltenen Varianten mit hoher Pathogenität, wie sie typischerweise bei monogenetischen Formen der Parkinson-Krankheit vorliegen, sind durch GenomeWide Association Studies (GWAS) häufige genetische Varianten getreten. Nach einem Fall-Kontroll-Prinzip werden dabei, über das ganze Genom verteilt, einzelne Nukleotid-Polymorphismen (auch „single nucleotide polymorphism“ [SNPs] genannt) verglichen. Eine signifikante Häufung bei Betroffenen mit einem bestimmten Phänotyp wird als assoziiert betrachtet. Vorteilhaft ist hier die nichthypothesenbasierte Herangehensweise, allerdings ist eine kausale Bedeutung der häufigen Variante selbst für die Erkrankung unwahrscheinlich, und selbst das zugrunde liegende Gen ist nicht sicher bestimmbar [34].

Aufgrund ihres seltenen Auftretens werden die monogenetischen Formen durch GWAS-Analysen typischerweise nicht abgebildet, allerdings weisen die Befunde der GWAS hin, dass die 
gleichen Gene, in denen seltene Mutationen eine seltene monogenetische Form der Parkinson-Krankheit verursachen, auch häufige Varianten tragen können, die ein erhöhtes Risiko für die Entwicklung der sporadischen Parkinson-Krankheit beitragen [29, 35]. Die Validierung genetischer Faktoren in der Pathogenese der Parkinson-Krankheit kann klassisch im Tiermodell (transgen, Knock-In/-Out) untersucht werden, aber zunehmend auch in von Patienten gewonnenem Material, welches in induzierte pluripotente Stammzellen programmiert werden kann. So können dopaminerge Neurone der Substantia nigra für einzelne Patienten und Mutation nachgebildet und untersucht werden [36].

\section{Genetische Stratifizierung - Lernen von monogenetischen Subtypen}

Exemplarisch für die monogenetischen Parkinson-Subtypen sowie Varianten mit erhöhtem Erkrankungsrisiko möchten wir aufgrund ihrer Häufigkeit im Folgenden LRRK2 (monogenetische Ursache) und GBA (häufigstes Risikogen) vorstellen, die auch im Hinblick auf die Entwicklung innovativer kausaler Therapien von großem Interesse für die Präzisionsmedizin sind.

\section{LRRK2}

Im Leucine-rich-repeat kinase 2 Gen (LRRK2) wurden zunächst Mutationen in Familien mit autosomal-dominant vererbter Parkinson-Krankheit festgestellt. In der Folge stellte sich eine weite Verbreitung einiger Varianten heraus, mit Auftreten sowohl bei sporadischen als auch familiären Formen weltweit [32, 37, 38]. Von den über 80 bekannten Varianten im LRRK2-Gen konnten 7 als pathogenetisch relevant bestätigt werden [39]. Neben Mutationen, die für die autosomal-dominante Vererbung in Familien verantwortlich sind, gibt es aber auch viele häufige genetische Varianten, die das Erkrankungsrisiko erhöhen bzw. erniedrigen (protektiv [40]). LRRK2-G2019 S-Parkinson wurde ein milderer Verlauf mit langsamerer Verschlechterung der motorischen Scores und weniger kognitive Einschränkungen sowie eine bessere olfaktorische Funktion zugeschrieben. In Bezug auf den motorischen Typ wird bei LRRK2-G2019 S zum einen ein tremor-dominanter Subtyp, zum anderen PIGD-Subtyp (PIGD = Postural Instability Gait Difficulty) als dominant beschrieben [41].

Die G2019 S-Mutation ist bei 4\% aller familiären und 1\% aller sporadischen Erkrankungen bei Kaukasiern in Europa vorhanden, in Nordafrika ist sie die Krankheitsursache bei über 30\% der Parkinson-Patienten, allerdings ist sie auch bei Gesunden nachweisbar [42]. In eine Studie von 2014 wurden tunesische und norwegische LRRK2 G2019 S-Mutationsträger mit sporadischer Parkinson-Krankheit und bislang Nicht-Betroffene im Hinblick auf die Penetranz verglichen. Dabei zeigte sich für sporadische Parkinson-Patienten ohne LRRK2-Mutation eine vergleichbare Verteilung des Erkrankungsbeginns in beiden Populationen, während die LRRK2 p.G2019 S-Patienten in Norwegen zu einem im Median 10 Jahre späteren Erkrankungsbeginn im Vergleich zu Patienten aus Tunesien führt [43]. Diese Befunde weisen deutlich auf populationsspezifische Faktoren in der Krankheitsausprägung hin, wobei neben dem unterschiedlichen genetischen Hintergrund (protektive und Risiko-Varianten in LRRK2 selbst oder in anderen Genen) auch unterschiedliche Lebensstilfaktoren (Ernährung) sowie Umweltfaktoren (Pestizide) eine Rolle spielen können [44].
LRRK2 interagiert als Kinase mit vielen in der Pathogenese der Parkinson-Krankheit implizierten Proteinen und scheint in verschiedene, im Rahmen der Neurodegeneration relevante Prozessen wie Dysfunktionen der Nervenzellen und des Immunsystems involviert zu sein $[45,46]$. Für die häufigste Mutation im LRRK2-Gen bei Kaukasiern, G2019 S, konnte früh eine pathogenetisch bedeutsame erhöhte Kinase-Aktivität nachgewiesen werden, was für einen „Gain-of-function“-Mechanismus der Mutation spricht [47, 48].

Tatsächlich konnte anhand patientenbasierter Stammzellmodelle gezeigt werden, dass Nervenzellen von Mutationsträgern der G2019 S-Mutation im LRRK2-Gen ein vermindertes Auswachsen von Nervenzell-Fortsätzen aufweisen und dass diese Störung direkt von der erhöhten Kinase-Aktivität abhängt [49]. Sowohl das genetische Korrigieren der Mutation in Zellen von Patienten als auch die Behandlung der Nervenzellen mit einem Kinase-Inhibitor waren in der Lage, das Auswachsen der Nervenzellen zu normalisieren [49]. Aufgrund dieser und weiterer Laborbefunde ist es inzwischen gelungen, Medikamente zu entwickeln, die die Kinase-Aktivität von LRRK2 hemmen und wichtige Sicherheitsaspekte in klinischen Phase-I-Studien erfüllen [50]. Aktuell werden erste klinische Studien bei betroffenen G2019 S-Mutationsträgern im LRRK2-Gen geplant, was die Umsetzung eines präzisionsmedizinischen, auf genetischer Stratifizierung basierenden Ansatzes impliziert [51]. Zwar wurde die G2019 S-Mutation nicht bei anderen Ethnien gefunden, aber in der indischen Bevölkerung wurde eine andere Mutation entdeckt, welche ebenfalls die Kinase-Aktivität von LRRK2 erhöht, sodass ein erfolgreich entwickelter Kinase-Inhibitor möglicherweise auch weiteren Patienten zugutekommen kann [52].

\section{GBA}

Die Glucocerebrosidase (GCase) ist ein lysosomales Enzym, das initial als homozygot mutiert beim Morbus Gaucher beschrieben wurde. Dabei handelt es sich um eine kindliche lysosomale Speicherkrankheit mit autosomal-rezessiver Vererbung, wobei der Enzymdefekt zu einer Akkumulation von Glucosylceramid und Glucosylsphingosin führt und diese Substrate daher in Lysosomen von Makrophagen akkumulieren [53]. Der Zusammenhang von GBA-Mutationen und Parkinson ergab sich zunächst aus FallBeobachtungen, die eine Häufung der Erkrankung in Familien mit M. Gaucher deutlich machten. Dieser Zusammenhang wurde letztlich in einer großen multizentrischen genetischen Assoziations-Studie nachgewiesen [54].

Während Patienten mit M. Gaucher (homozygote Mutationsträger) Zeichen von Parkinsonismus aufweisen, sind bei der sporadischen Parkinson-Krankheit typischerweise die heterozygoten GBA-Mutationsträger betroffen. Hierbei wird ebenfalls eine pathogenetische Rolle der eingeschränkten GCase-Funktion für die Neurodegeneration angenommen. Dabei zeigt sich, dass es durch durch die gestörte lysosomale Funktion zu einer Störung des autophagischen Abbaus von $\alpha$-Synuclein mit nachfolgender Akkumulation im Sinne eines Circulus vitiosus kommt [55].

Klinisch zeigen GBA-PD-Patienten einen im Vergleich zur typischen idiopathischen Parkinson-Krankheit mit ca. 4 Jahren früheren Erkrankungsbeginn, zudem sind Ruhetremor und Bradykinesie weniger ausgeprägt, wohingegen kognitive Einschränkungen und axiale Symptome deutlich häufiger anzutreffen sind [41, 54, 56]. Passend zu den vermehrten kognitiven 
Störungen bestehen eine Assoziation mit der Demenz mit LewyKörpern (DLB) und ein häufigeres Auftreten von REM-Schlaf-Verhaltensstörungen (RBD) [41]. Die Parkinson-assoziierten Mutationen werden als Risikofaktoren gewertet und sind für bis zu $10 \%$ aller sporadischen Parkinson-Fälle verantwortlich [57].

Neben GBA scheinen auch weitere Varianten in Genen, die mit anderen lysosomalen Speicherkrankheiten assoziiert sind, eine Rolle in der Pathogenese der Parkinson-Krankheit zu spielen [58]. Dabei ergeben sich Hinweise für eine Akkumulation von Risikovarianten in verschiedenen Genen des lysosomalen Abbauwegs als Ursache für die Parkinson-Krankheit bei einer Subgruppe der Patienten [58], sodass die Hoffnung besteht, dass Medikamente, die die lysosomale Funktion bei GBA-Mutationsträgern verbessern, auch bei weiteren Patienten therapeutisch wirksam sein könnten.

Basierend auf den bei M. Gaucher eingesetzten Therapien der intravenösen Enzymersatztherapie (ERT), die allerdings nicht die Blut-Hirn-Schranke passiert, und der Substratreduktionstherapien (SRT), werden auch bei GBA-assoziierter Parkinson-Krankheit SRTs mit Liquorgängigkeit erprobt [55]. Aktuell wird u.a. Venglustat per os von Sanofi multizentrisch getestet [59, 60]. Die SRT zielt auf eine reduzierte Ablagerung der Lipidsubstrate ab, durch eine Reduktion ihrer Biosynthese [48].

Eine andere Herangehensweise ist der Einsatz von chemischen Chaperonen, die die fehlgefalteten Proteine stabilisieren bzw. neu falten. Vorteilhaft ist die mögliche Passage der Blut-HirnSchranke durch die kleine Größe der Chaperone [55]. Bei einem Screening von bekannten Wirkstoffen auf eine Wirksamkeit auf GCase wurde das Mucolytikum Ambroxol als aktivitätssteigernd identifiziert [61] und in der Folge in Fibroblasten von gesunden Kontrollen, Patienten mit M. Gaucher und heterozygoten Mutationsträgern mit und ohne Parkinson eine Steigerung der Aktivität und Reduktion von oxidativem Stress nachgewiesen [62]. Darüber hinaus konnte mithilfe eines Gcase-Modulators in durch aus induzierten pluripotenten Stammzellen (iPSC) gewonnenen mesencephalen, dopaminergen Neuronen von Trägern verschiedener Parkinson-assoziierter Mutationen gezeigt werden, dass die alleinige Steigerung der Aktivität der GCase zur Reduktion der GCase-Substraten und Clearance von $\alpha$-Synuclein führte und damit u.a. die lysosomale Funktion verbesserte [63]. Die aktuelle AiM-PD [64] untersuchte diesen Zusammenhang in jeweils 10 Patienten mit und ohne GBA1-Mutation in einer Phase-II-Studie, dabei konnten eine reduzierte Liquor-GCase-Aktivität sowie eine erhöte Liquor- $\alpha$-Synuclein-Konzentration bei den 18 Patienten festgestellt werden, die die Studie abgeschlossen hatten.

Für den zunächst im Parkinson-/M. Gaucher-Tiermodell getesteten AAV-GBA1-Vektor (AAV = adeno-associated viral infection), der mittels „Infektion“ korrektes Gen-Material in Chromosom 19 integriert, werden nun für eine Studie der Firma „Prevail Therapeutics“ erstmalig 16 PD-GBA Patienten für eine Studie mit einmaliger intracisternaler Injektion und 5-Jahre-Follow-Up rekrutiert [65].

Somit konnten durch die Erforschung der monogenetischen Parkinson-Formen erstmals betroffene molekulare Signalwege der Neurodegeneration erkannt und dadurch spezifische kausale Therapien definiert werden, sodass eine berechtigte Hoffnung besteht, dass die Ergebnisse zumindest auf Untergruppen der häufigen idiopathischen Form der Erkrankung übertragen werden können.
Ein weiterer Fortschritt ergab sich durch patientenbasierte zelluläre Modelle, die z.B. auf induzierten pluripotenten Stammzellen (iPSC) beruhen, die aus per Hautbiopsie gewonnenen Fibroblasten differenziert werden können [66] oder auf den aus iPSC generierten Mini-Gehirnen - 3-D-Modelle, die gewebespezifische Zellkompositionen nachstellen, wie z.B. als mesencephales Organoid [67]. Automatisierungsplattformen können in der Folge eingesetzt werden, um in kurzer Zeit eine Vielzahl von Substanzen mit den oben genannten Modellen auf ihre Wirksamkeit zu testen und damit zur Findung neuer Therapien beitragen [68].

\section{Pharmakogenetik}

\section{Symptomatische dopaminerge Therapien - Re-Evaluierung aktueller Therapien}

Variables Ansprechen auf und Nebenwirkungen von Therapien sind in der klinischen Medizin weit verbreitet und können teilweise durch genetische Unterschiede erklärt werden, z.B. die Wirksamkeit von Azathioprin in Abhängigkeit von genetischen Polymorphismen bei chronisch entzündlichen Darmerkrankungen [69]. Hand in Hand mit kostengünstigeren Sequenzierungsmethoden konnten einige dieser genetischen Varianten identifiziert werden. Durch die Pharmakogenetik wird versucht, die Therapie zu individualisieren und richtig dosierte, nebenwirkungsfreie Therapien für den einzelnen Patienten zu finden [70].

Die medikamentöse Parkinson-Therapie ist nach wie vor eine symptomatische und zielt im Wesentlichen darauf ab, ein dopaminerges Defizit durch den zunehmenden Verlust der dopaminergen Neurone auszugleichen, ergänzt durch NMDA-antagonistische und anticholinerge Strategien [71]. Gold-Standard der Therapie ist dabei Levodopa als die Blut-Hirn-Schranke passierender Dopaminverläufer, zusammen mit einem peripheren Dopa-DecarboxylaseHemmer, ergänzt von Dopamin-Agonisten, COMT-Inibitoren (COMT = Catechol-o-methyltransferase) und MAO-B-Inhibitoren (MAO-B = Monoaminooxidase $\mathrm{B}$ ), welche in einem anderen Beitrag in dieser Ausgabe detailliert beschrieben werden.

Ihre Wirksamkeit und auch das Nebenwirkungsprofil variieren von Patient zu Patient, weshalb mit genetischen Screenings nach Ursachen gesucht wurde. Dies beruht auf der Annahme, dass es u.a. Unterschiede in der Rezeptorbindung, dem Dopamin-Metabolismus und dem Durchtritt durch die Blut-Hirn-Schranke verantwortlich für den variablen Behandlungserfolg sind bei Veränderungen der Rezeptoren/Enzyme/Transporter [72].

\section{Stratifizierung zur verbesserten symptomatischen Therapie}

Levodopa-Response

Ein ausgeprägter genetischer Effekt auf die Levodopa-Pharmakokinetik konnte bisher nicht festgestellt werden. In einigen Studien wurde ein Zusammenhang von Polymorphismen im SLC6A3-Gen sowie im Dopa-Decarboxylase-Gen (DDC oder auch AADC-Aromatische-L-Aminosäure-Decarboxylase) auf die motorische Levodopa-Sofortantwort (erhöht/erniedrigt) postuliert, wohingegen Veränderungen im COMT- und MAOB-Genen hingegen eher mit 
einer veränderten Wirkung von Langzeitdosen von Levodopa assoziiert seien [72].

\section{DR2 + Rasagiline}

Die größte Studie bei Parkinson-Patienten im Frühstadium, unter einer Monotherapie mit dem MAO-B-Hemmer Rasagilin, zeigte erstmals Hinweise auf ein differenzielles Ansprechen von Patienten entsprechend der genetischen Prädisposition. Bei den Untersuchungen von genetischen Faktoren, die das Ansprechen der Patienten auf die Therapie mit Rasagilin und die Krankheitsprogression unter dieser Therapie beeinflussen, wurden Polymorphismen in 20 Kandidaten-Genen ausgewählt, die mit der Pharmakokinetik von Rasagilin in Zusammenhang stehen oder mit einem erhöhten Risiko, an Parkinson zu erkranken, assoziiert sind [73]. Dabei konnten die Autoren zeigen, dass zwei SNPs im Dopamin-D2-Rezeptor-Gen (DRD2) mit einem besseren Therapieansprechen bei Teilnehmern der ADAGIO-Studie (Einschluss von 629 Patienten aus der Rasagilin-Gruppe) verbunden sind [74]. Dieses Ansprechen war jedoch nicht mit einem postulierten krankheitsverlaufsmodulierenden Effekt von Rasagilin assoziiert.

\section{COMT}

Im COMT-Gen wurden verschiedene Polymorphismen beschrieben, die Einfluss auf das Ansprechen von Parkinson-Patienten auf eine Therapie mit Levodopa haben. Der rs4680-Polymorphismus ist mit einer erniedrigten Enzymaktivität assoziiert und wurde in mehreren Kohorten untersucht [75]. In einer polnischen Studie [76] wurde festgestellt, dass die Tagesdosis der Levodopa-Medikation nach 5 Therapiejahren in Zusammenhang mit der jeweiligen Enzymaktivität des funktionalen COMT-Haplotyps erhöht ist (niedrig < mittel < hoch).

\section{Nebenwirkungen}

Motorische Komplikationen: Motorische Fluktuationen und Dyskinesien

Levodopa-induzierte Dyskinesien, also unwillkürliche Bewegungen, treten häufig im Rahmen von langjähriger Levodopa-Therapie auf. Das (frühe) Auftreten hängt möglicherweise auch von genetischen Faktoren ab, dabei wurden Assoziationen von bestimmten genetischen Varianten im Dopamin-Rezeptor 2/3 (DRD2 und DRD3) sowie im Dopamin-Transporter Typ I und auch SLC6A3-Gen (SLC6A3 = Solute Carrier Family 6, member 3) (Neurotransmitter-Transporter) beschrieben [72].

Es besteht also ein Zusammenhang zwischen dem Therapieansprechen bzw. möglichen Nebenwirkungen von dopaminerger Medikation und Polymorphismen in den entsprechenden Enzymund Rezeptor-kodierenden Genen. Die derzeitigen Studienergebnisse reichen allerdings nicht aus, um Konsequenzen für den klinischen Alltag zu ziehen. Weitere Replikations- und Validationsstudien und das Einbeziehen von größeren Kohorten sind nötig.

\section{Tiefe Hirnstimulation (THS)}

Ein systematischer Review von THS bei monogenetischen Parkinson-Formen von Kuusimäki et al. (2019) untersuchte Daten aus 46 Studien mit insgesamt 221 Patienten mit genetischem Parkinson [77] und jeweils kleinen Fallzahlen. Insgesamt ergab sich für
Träger einer Mutation im LRRK2-Gen (vor allem G2019 S) und homozygote Parkin-Mutationsträger ein guter Therapie-Effekt auf die motorischen Symptome. Eine Stratifikation, basierend auf den verschiedenen Stimulationsorten (Globus pallidus internus bzw. Nucleus subthalamicus), erfolgte aufgrund der geringen Fallzahlen allerdings nicht.

Demgegenüber scheinen Patienten, die eine Mutation im GBAGen tragen, insgesamt ein schlechteres Ansprechen auf die Tiefe Hirnstimulation im Nucleus subthalamicus aufzuweisen - im Vergleich zu sporadischen Parkinson-Patienten [78]. Hervorzuheben ist dabei vor allem die Relevanz der neuropsychiatrischen und kognitiven Symptome der einzelnen Subtypen, wie bei GBA, SNCA. Aber auch in einer LRRK2-Variante (p.T2031 S) zeigten sich neuropsychiatrische Auffälligkeiten bei GBA-Mutationsträgern, ein rasch progredienter kognitiver Abbau und Depression und bei SNCA (und einer LRRK2-Variante) Depression und Psychose. Ein weiterer Review von 2019 [79] kam zu ähnlichen Ergebnissen, bewertet die klinische Anwendbarkeit der Ergebnisse allerdings zurückhaltender und sieht die Relevanz eher in der Abschätzung der Prognose (schneller kognitiver Abbau bei GBA).

Wie wichtig eine Abgrenzung von atypischen PD ist, zeigte ein Review von THS in Patienten mit langsam progredienter Multisystematrophie (MSA), die sich nach einer teils initialen Verbesserung stark verschlechterten [80]. Aktuell können keine sicheren Empfehlungen für oder gegen die THS aufgrund des genetischen Profils und PD-Subtypen ausgesprochen werden, da insbesondere die Fallzahlen zu klein sind und andere Einflussfaktoren, vor allem eine variable Elektrodenplatzierung, eine Rolle für den Therapieerfolg spielen können. In der Zukunft kann das Wissen über die einzelnen Subtypen mit Fokus auf dem Krankheitsverlauf zusätzliche Informationen für eine Therapieentscheidung bieten. Wichtiger scheint aktuell die präoperative Evaluation von NMS mit Wirkung auf die postoperative Lebensqualität [81] und das Outcome, wobei die Wirkungen auf die NMS auch hier mit der Positionierung der Elektroden bzw. der Stimulationslokalisation zusammenhängen [82].

\section{Re-Evaluation der prodromalen Phase}

Mit einer verbesserten Stratifizierung von klinischen Subtypen und der Identifikation von beeinträchtigten Stoffwechselwegen geht der Wunsch einher, durch neuroprotektive Therapien den Verlauf zu verlangsamen und, wenn möglich, das Vollbild der Erkrankung zu verhindern.

Mit diesem Ziel vor Augen müssen auch die Erkennung und die Einordnung von prodromalen Stadien möglich werden, gerade weil für die prodromalen Symptome unterschiedliche Latenzzeiten bis zur manifesten Diagnose bekannt sind. Dies bedeutet allerdings nicht, dass es bei Vorliegen eines ProdromalStadiums definitiv zu einer Phänokonversion kommt.

Auch wenn die Parkinson-Krankheit mit einer Prävalenz von $1 \%$ bei Menschen über 60 Jahren relativ häufig ist, ist es unrealistisch, zum Studium prämotorischer Krankheitsphasen eine große Anzahl von Probanden in populationsbasierten Kohorten über einen langen Zeitraum zu verfolgen, sodass Probanden aufgrund bestimmter prodromaler Symptome präselektiert werden müssen, die das Risiko, zu erkranken, erhöhen. 
Eine Möglichkeit, die vielfach genutzt wird, ist die Verfolgung von asymptomatischen Trägern von genetischen Mutationen, insbesondere GBA und LRRK2, da in diesen Fällen ausreichend große Kohorten möglich sind. Alternativ können Patienten mit REMSchlaf-Verhaltensstörungen (RBD) untersucht werden, die eine über 80\%ige Wahrscheinlichkeit aufweisen, innerhalb von 10 Jahren die Parkinson-Krankheit zu entwickeln [83]. Bei allen Vorteilen einer „angereicherten“ Risiko-Kohorte, die auf dem Vorliegen von RBD oder einer GBA/LRRK2-Mutation beruht, muss beachtet werden, dass Erkenntnisse über diese Prodromal Carrier nicht auf alle PD-Patienten übertragen werden können. So entwickelt sich zwar die große Mehrzahl der RBD-Patienten über einen 10-jährigen Verlauf eine Synukleinopathie, wie iPD, Multisystematrophie (MSA) oder Demenz mit Lewy-Körper (DLB) auf, jedoch liegt bei weitem nicht bei jedem PD-Patienten RBD vor. In der Gesamtgruppe der Parkinson-Patienten weist nur knapp die Hälfte im Krankheitsverlauf ein RBD auf [84, 85].

Als Alternative bieten sich Ansätze an wie die bei der PREDICT-PD-Studie eingesetzte Risikokalkulation - basierend auf einer Assoziation verschiedener bekannter Risikofaktoren und einem bevölkerungsbasiertem Screening mit Online-Tools (Fragebögen, Tastatur-Bradykinesie-Test) und per Post versendeter Assessments (Riech-Test, DNA-Saliva-Kit) mit Follow-Up [86]. Auch die Movement Disorders Society (MDS) hat ihrem Update der "research criteria for prodromal $P D^{\text {“ }}$ einen Risikorechner zur Seite gestellt $[87,88]$. Mit der Nutzung von Online-Tools werden bevölkerungsbasierte Ansätze mit großen Kohorten machbar, darüber hinaus vereinfachen einheitliche Datenerhebungssysteme, z.B. das Redcap-System, internationale Kooperationen und Datenabgleich [89, 90].

Obwohl die Genotypisierung weitaus günstiger geworden ist, muss der weitläufige Einsatz kritisch evaluiert werden, da das Wissen um das Risiko, an einer neurodegenerativen Erkrankung zu erkranken, im Hinblick auf noch nicht hinreichend verstandene Mechanismen der Penetranz, der klinischen Heterogenität und den Mangel an kausalen Therapien eine große Belastung für Carrier darstellen kann - mit Konsequenzen auf Lebensführung und -qualität.

Für eine sichere Früherkennung und Therapieentscheidungen bei Personen, die kurz vor der Phänokonversion stehen, brauchen wir Biomarker.

Biomarker sind objektive, quantifizierbare Parameter, die als Indikatoren biologischer und pathogener Prozesse erhoben werden können [91]. Dabei kann man sie verschiedenen Kategorien zuordnen, so können sie prädiktiv, (differenzial-)diagnostisch, prognostisch oder arzneimittelbezogen interpretiert werden. Klassische biochemische Parameter sind bestimmbar in Körperflüssigkeiten (sog. Liquid biomarkers) - bei PD u.a. in Speichel, Blut und Liquor - sowie in festen Geweben (sog. Solid biomarkers) wie in Kolonbiopsien, Speicheldrüsenbiopsien oder Hautbiopsien. $\alpha$-Synuclein wird dabei sowohl in Körperflüssigkeiten als auch in Gewebeproben untersucht. Dazu kommen objektivierbare Messwerte aus sensorbasierten Untersuchungen (z.B. Messung von Handbewegungen mittels Bewegungssensoren) oder Bildgebungsparameter (z.B. SPECT) [92].

Bei der Suche danach ergaben sich, auch bedingt durch die klinische Heterogenität und den langen Verlauf, bisher keine sicheren biochemischen Einzel-Biomarker in den Körperflüssigkeiten und Geweben. Mögliche Biomarker gibt es im Kontext von mitochondrialer Dysfunktion, oxidativem Stress, Lewy-Körper-Formation und Neuroinflammation, dabei ist die Kombination von mehreren Biomarker-Kandidaten am aussichtsreichsten [22, 93].

Zusammenfassend ergeben sich aktuell wesentliche neue Ansätze zur Stratifizierung der Parkinson-Krankheit mit Optionen für neue präzisionsmedizinische Ansätze, aufbauend auf einer mechanismenbasierten Gruppierung von Parkinson-Patienten. Hier werden die Ergebnisse von aktuellen Studien mit potenziell neuroprotektiven Substanzen bei Trägern von GBA- und LRRK2Mutationen wegweisend für dieses Konzept. Sollte sich dieses Konzept bestätigen, wäre gegebenenfalls auch zu erwägen, vormals untersuchte potenziell neuroprotektive Substanzen wie Rasagilin oder auch Coenzym Q10 in entsprechend stratifizierten Subgruppen der Parkinson-Krankheit, basierend auf RezeptorPolymorphismen oder mitochondrialer Suszeptibilität, erneut zu evaluieren. Gleichfalls ergeben sich zunehmend Stratifizierungsmöglichkeiten für symptomatische Therapie-Ansätze bei der Parkinson-Krankheit, sodass sich insgesamt neue Optionen für die Verbesserung von Therapie-Optionen für diese häufige neurodegenerative Erkrankung ergeben.

\section{Interessenkonflikt}

1. Rejko Krüger (RK) dient als Editorial Board Member des European Journal of Clinical Investigation, des Journal of Parkinsonism and Related Disorders and des Journal of Neural Transmission. RK hat Forschungsförderung vom Fonds National de Recherche de Luxembourg (FNR), als Coordinator des National Centre for Excellence in Research on Parkinson's disease (NCER-PD) und innerhalb des PEARL Excellence Programme [FNR/P13/6682797/Krüger], sowie für folgende Projekte MiRisk [C17/BM/11676395], MotaSYN [12719684], MAMaSyn ). RKs Arbeit wird von der Deutschen Forschungsgemeinschaft (DFG; KR2119/ 8-1), der Michael J Fox Foundation, sowie des European Union's Horizon2020 research and innovation program (WIDESPREAD; CENTRE-PD grant agreement no. 692320) gefördert. RK hat Referentenhonorare und/oder Reisekostenförderung von Abbvie, Zambon und Medtronic erhalten und er nimmt als PI oder site-PI an industriegeförderten Projekten teil, ohne Honorare zu erhalten.

2. Lara Stute hat Reisekostenförderung durch Zambon erhalten.

\section{Literatur}

[1] Love-Koh J, Peel A, Rejon-Parrilla JC et al. The future of precision medicine: potential impacts for health technology assessment. Pharmacoeconomics. 2018; 36: 1439-1451. Im Internet: https://doi.org/10.1007/ s40273-018-0686-6

[2] Le Tourneau C, Kamal M, Bièche I. Precision medicine in oncology: what is it exactly and where are we? Per Med 2018; 15: 351-353. Im Internet: https://doi.org/10.2217/pme-2018-0036; Stand: 29/01/2020

[3] Renfro LA, Sargent DJ. Statistical controversies in clinical research: basket trials, umbrella trials, and other master protocols : A review and examples. Special Article 2017; 34-43

[4] Wimo A, Jönsson L, Gustavsson A Cost of illness and burden of dementia - The base option. Im Internet: https://www.alzheimer-europe.org/Res earch/European-Collaboration-on-Dementia/Cost-of-dementia/Cost-ofillness-and-burden-of-dementia ; Stand: 29/01/2020

[5] Why choose neurodegenerative diseases? Im Internet: https://www.ne urodegenerationresearch.eu/about/why/ Stand: 29/01/2020 
[6] Nerius M, Fink A, Doblhammer G. Parkinson's disease in Germany: Prevalence and incidence based on health claims data. Acta Neurol Scand 2017; 136: 386-392

[7] Ray Dorsey E, Elbaz A, Nichols E et al. Global, regional, and national burden of Parkinson's disease, 1990-2016: a systematic analysis for the global burden of disease study 2016. Lancet Neurol 2018; 17: 939-953

[8] Nutt JG. Motor subtype in Parkinson's disease: different disorders or different stages of disease? Mov Disord 2016; 31: 957-961

[9] Selikhova M, Williams DR, Kempster PA et al. A clinico-pathological study of subtypes in Parkinson's disease. Brain 2009; 132: 2947-2957

[10] Pagano G, Ferrara N, Brooks DJ et al. Age at onset and Parkinson disease phenotype. Neurology 2016; 86: 1400-1407

[11] Paisán-Ruiz C. Young-onset Parkinson disease. Im Internet: https://ww w.orpha.net/consor/cgi-bin/OC_Exp.php?Lng=EN\&Expert=2828 Stand: 29/01/2020

[12] Schrag A, Schott JM. Epidemiological, clinical, and genetic characteristics of early-onset parkinsonism. Lancet Neurol 2006; 5: 355-363

[13] Hoehn MM, Yahr MD. Parkinsonism: onset, progression, and mortality. Neurology 1967: 17 427-442

[14] Goetz CG, Poewe W, Rascol O et al. Movement disorder society task force report on the Hoehn and Yahr staging scale: status and recommendations. Mov Disord 2004; 19: 1020-1028

[15] Movement disorder society task force on rating scales for Parkinson's disease. The Unified Parkinson's Disease Rating Scale (UPDRS): status and Recommendations Movement. 2003; 18: 738-750

[16] Martinez-Martin P, Rodriguez-Blazquez C, Alvarez-Sanchez M et al. Expanded and independent validation of the movement disorder society-unified Parkinson's disease rating scale (MDS-UPDRS). J Neurol 2013; 260: 228-236

[17] Chaudhuri KR, Sauerbier A, Rojo JM et al. The burden of non-motor symptoms in Parkinson's disease using a self-completed non-motor questionnaire: A simple grading system. Park Relat Disord 2015; 21: 287-291

[18] Carrozzino D. Clinimetric approach to rating scales for the assessment of apathy in Parkinson's disease: a systematic review. Prog Neuro-Psychopharmacology Biol Psychiatry 2019; 94: 109641

[19] Sauerbier A, Jenner P, Todorova A et al. Non motor subtypes and Parkinson's disease. Park Relat Disord 2016; 22: S41-S46

[20] Di Battista ME, Cova I, Rubino A et al. Intercepting Parkinson disease non-motor subtypes: a proof-of-principle study in a clinical setting. J Neurol Sci 2018; 388: 186-191

[21] Mu J, Chaudhuri KR, Bielza C et al. Parkinson's disease subtypes identified from cluster analysis of motor and non-motor symptoms. Front Aging Neurosci 2017; 9: 1-10

[22] He R, Yan X, Guo J et al. Recent advances in biomarkers for Parkinson's disease. Front Aging Neurosci 2018; 10: 1-19

[23] Liu G, Locascio J], Corvol J-C et al. Prediction of cognition in Parkinson's disease with a clinical-genetic score: A longitudinal analysis of nine cohorts. Lancet Neurol 2017; 16: 620-629

[24] Sardi SP, Simuni T. New era in disease modification in Parkinson's disease: review of genetically targeted therapeutics. Park Relat Disord 2019; 59: 32-38

[25] Noyce A], Bandres-ciga S, Kim J et al. The Parkinson's Disease Mendelian Randomization Research Portal. Mov Disord 2019; 34: 1864-1872 Im Internet: http://dx.doi.org/10.1101/604033

[26] Heinzel S, Lerche S, Maetzler W et al. Global, yet incomplete overview of Cohort studies in Parkinson's disease. J Parkinsons Dis 2017; 7: 423-432

[27] Pringsheim T, Jette N, Frolkis A et al. The prevalence of Parkinson's disease: a systematic review and meta-analysis. Mov Disord 2014; 29 : 1583-1590

[28] Burbulla LF, Krüger R. Converging environmental and genetic pathways in the pathogenesis of Parkinson's disease. J Neurol Sci 2011; 306: 1-8
[29] Reed X, Bandrés-Ciga S, Blauwendraat $C$ et al. The role of monogenic genes in idiopathic Parkinson's disease. Neurobiol Dis 2019; 124: 230-239

[30] Blauwendraat C, Nalls MA, Singleton AB. The genetic architecture of Parkinson's disease. Lancet Neurol 2019; 4422: 1-9

[31] Tan MMX, Malek N, Lawton MA et al. Genetic analysis of Mendelian mutations in a large UK population-based Parkinson's disease study. Brain 2019; 142: 2828-2844

[32] Hentati F, Thompson C, Nosova E et al. LRRK2 parkinsonism in Tunisia and Norway: a comparative analysis of disease penetrance. Neurology 2014: 83 (6) 568-569

[33] Blauwendraat C, Faghri F, Pihlstrom L et al. NeuroChip, an updated version of the NeuroX genotyping platform to rapidly screen for variants associated with neurological diseases. Neurobiol Aging 2017; 57: 247. e9-247.e13

[34] Visscher PM, Wray NR, Zhang Q et al. 10 years of GWAS discovery: biology, function, and translation. Am J Hum Genet 2017; 101: 5-22

[35] Krüger R. Genes in familial parkinsonism and their role in sporadic Parkinson's disease. J Neurol Suppl 2004; 251: 2-6

[36] Finkbeiner S. Skibinski. In: Drug discovery in Parkinson's disease: update and developments in the use of cellular models. Int J High Throughput Screen 2011; 2011: 15

[37] Lesage S, Brice A. Role of mendelian genes in „sporadic“ Parkinson's disease. Park Relat Disord 2012; 18: S66-S70

[38] Heckman MG, Soto-Ortolaza Al, Aasly JO et al. Population-specific frequencies for LRRK2 susceptibility variants in the genetic epidemiology of Parkinson's disease (GEO-PD) consortium. Mov Disord 2013; 28: $1740-1744$

[39] Domingo A, Klein C. Genetics of Parkinson disease. Vol. 1. Aufl. Elsevier B.V. Amsterdam, Netherlands; 2018

[40] Ross OA, Soto-Ortolaza Al, Heckman MG et al. Association of LRRK2 exonic variants with susceptibility to Parkinson's disease: a case-control study. Lancet Neurol 2011; 10: 898-908

[41] Giladi N, Mirelman A, Thaler A et al. A personalized approach to Parkinson's disease patients based on founder mutation analysis. Front Neurol 2016; 7: 1-5

[42] Healy DG, Falchi M, O'Sullivan SS et al. Phenotype, genotype, and worldwide genetic penetrance of LRRK2-associated Parkinson's disease: a case-control study. Lancet Neurol 2008; 7: 583-590

[43] Hentati F, Trinh J, Thompson C et al. LRRK2 parkinsonism in Tunisia and Norway: a comparative analysis of disease penetrance. Neurology 2014; 83: 568 LP-569

[44] Krüger R. LRRK2 in Parkinson's disease - Drawing the curtain of penetrance: A commentary. BMC Med 2008; 6: 4-7

[45] Herbst S, Gutierrez MG. LRRK2 in infection: friend or foe? ACS Infect Dis 2019; 5: 809-815

[46] Derkinderen P, Neunlist M. Crohn's and Parkinson disease: Is LRRK2 lurking around the corner? Nat Rev Gastroenterol Hepatol 2018; 15: 330-331

[47] Gloeckner C], Kinkl N, Schumacher A et al. The Parkinson disease causing LRRK2 mutation I2020T is associated with increased kinase activity. Hum Mol Genet 2006; 15: 223-232

[48] Sardi SP, Cedarbaum JM, Brundin P. Targeted therapies for Parkinson's disease: from genetics to the clinic. Mov Disord 2018; 33: 684-696

[49] Reinhardt P, Schmid B, Burbulla LF et al. Genetic correction of a Irrk2 mutation in human iPSCs links parkinsonian neurodegeneration to ERK-dependent changes in gene expression. Cell Stem Cell 2013; 12: 354-367

[50] Study to evaluate DNL201 in subjects with Parkinson's Disease. Im Internet: https://clinicaltrials.gov/ct2/show/NCT04056689?term=Denali\& cond=Parkinson $\& d r a w=2$ Stand: $29 / 01 / 2020$

[51] Brüggemann N, Klein C. Will genotype drive treatment options? Mov Disord 2019; 34:1864-1872 
[52] Kishore A, Ashok Kumar Sreelatha A, Sturm M et al. Understanding the role of genetic variability in LRRK2 in Indian population. Mov Disord 2019; 34: 496-505

[53] Nalysnyk L, Rotella P, Simeone JC et al. Gaucher disease epidemiology and natural history: a comprehensive review of the literature. Hematology 2017; 22: 65-73

[54] Sidransky E, Nalls MA, Aasly JO et al. Multicenter analysis of glucocerebrosidase mutations in Parkinson's disease. N Engl J Med 2009; 361: 1651-1661

[55] Do J, McKinney C, Sharma P et al. Glucocerebrosidase and its relevance to Parkinson disease. Mol Neurodegener 2019; 14: 1-16

[56] Brockmann K, Srulijes K, Pflederer S et al. GBA-associated Parkinson's disease: reduced survival and more rapid progression in a prospective longitudinal study. Mov Disord 2015; 30: 407-411

[57] Sheerin UM, Houlden H, Wood NW. Advances in the genetics of Parkinson's disease: a guide for the clinician. Mov Disord Clin Pract 2014; 1: 3-13

[58] Robak LA, Jansen IE, van Rooij J et al. Excessive burden of lysosomal storage disorder gene variants in Parkinson's disease. Brain 2017; 140: 3191-3203

[59] Judith Peterschmitt M, Gasser T, Isaacson S et al. Safety, tolerability and pharmacokinetics of oral venglustat in Parkinson disease patients with a GBA mutation. Mol Genet Metab 2019; 126: S117

[60] A global study to assess the drug dynamics, efficacy, and safety of GZ/ SAR402671 in Parkinson's disease patients carrying a glucocerebrosidase (GBA) gene mutation (MOVES-PD). Im Internet: https://clinicaltri als.gov/ct2/show/NCT02906020. Stand: 29/01/2020

[61] Maegawa GHB, Tropak MB, Buttner JD et al. Identification and characterization of ambroxol as an enzyme enhancement agent for Gaucher disease. J Biol Chem 2009; 284: 23502-23516

[62] McNeill A, Magalhaes ], Shen C et al. Ambroxol improves lysosomal biochemistry in glucocerebrosidase mutation-linked Parkinson disease cells. Brain 2014; 137: 1481-1495

[63] Mazzulli JR, Zunke F, Tsunemi T et al. Activation of $\beta$-glucocerebrosidase reduces pathological $\alpha$-synuclein and restores lysosomal function in Parkinson's patient midbrain neurons. J Neurosci 2016; 36: 7693-7706

[64] Mullin S, Smith L, Lee K et al. Ambroxol for the Treatment of Patients with Parkinson Disease with and without Glucocerebrosidase Gene Mutations: a Nonrandomized, Noncontrolled Trial. JAMA Neurol 2020 77: 427

[65] Phase 1/2a clinical trial of PR001A in patients with Parkinson's disease with at least one GBA1 mutation (PROPEL). Im Internet: https://clinicalt rials.gov/ct2/show/study/NCT04127578?term=AAV\%2C+Prevail\&cond= Parkinson+Disease $\& d r a w=2 \& r a n k=1$ Stand: $29 / 01 / 2020$

[66] Zhou Q, Melton DA. Extreme makeover: converting one cell into another. Cell Stem Cell 2008; 3: 382-388

[67] Grenier K, Kao J, Diamandis P. Three-dimensional modeling of human neurodegeneration: Brain organoids coming of age. Mol Psychiatry 2019 25: 254-274

[68] Rocha DN, Carvalho ED, Pêgo AP. High-throughput platforms for the screening of new therapeutic targets for neurodegenerative diseases. Drug Discov Today 2016; 21: 1355-1366

[69] Stocco G, Pelin M, Franca R et al. Pharmacogenetics of azathioprine in inflammatory bowel disease: A role for glutathione-S-transferase? World J Gastroenterol 2014; 20: 3534-3541

[70] Roden DM, McLeod HL, Relling MV et al. Pharmacogenomics. Lancet 2019; 394: 521-532

[71] Ellis JM, Fell MJ. Current approaches to the treatment of Parkinson's disease. Bioorganic Med Chem Lett 2017; 27: 4247-4255

[72] Corvol JC, Poewe W. Pharmacogenetics of Parkinson's disease in clinical practice. Mov Disord Clin Pract 2017; 4: 173-180
[73] Masellis M, Collinson S, Freeman N et al. Dopamine D2 receptor gene variants and response to rasagiline in early Parkinson's disease: a pharmacogenetic study. Brain 2016; 139: 2050-2062

[74] Olanow CW, Hauser RA, Jankovic J et al. A randomized, double-blind, placebo-controlled, delayed start study to assess rasagiline as a disease modifying therapy in Parkinson's disease (the ADAGIO study): rationale, design, and baseline characteristics. Mov Disord 2008; 23: 2194-2201

[75] Politi C, Ciccacci C, Novelli G et al. Genetics and Treatment Response in Parkinson's Disease: an Update on Pharmacogenetic Studies. NeuroMolecular Med 2018: 20: 1-17

[76] Bialecka M, Kurzawski M, Klodowska-Duda G et al. The association of functional catechol-O-methyltransferase haplotypes with risk of Parkinson's disease, levodopa treatment response, and complications. Pharmacogenet Genomics 2008: 18: 815-821

[77] Kuusimäki T, Korpela J, Pekkonen E et al. Deep brain stimulation for monogenic Parkinson's disease: a systematic review. J Neurol 2019; 0: 0

[78] Weiss D, Brockmann K, Srulijes K et al. Long-term follow-up of subthalamic nucleus stimulation in glucocerebrosidase-associated Parkinson's disease. J Neurol 2012; 259: 1970-1972

[79] Ligaard J, Sannæs J, Pihlstrøm L. Deep brain stimulation and genetic variability in Parkinson's disease: a review of the literature. Npj Park Dis 2019; 5: 1-10

[80] Meissner WG, Laurencin C, Tranchant C et al. Outcome of deep brain stimulation in slowly progressive multiple system atrophy: a clinicopathological series and review of the literature. Park Relat Disord 2016; 24: 69-75

[81] Dafsari HS, Weiß L, Silverdale M et al. Short-term quality of life after subthalamic stimulation depends on non-motor symptoms in Parkinson's disease. Brain Stimul 2018; 11: 867-874

[82] Petry-Schmelzer JN, Krause M, Dembek TA et al. Non-motor outcomes depend on location of neurostimulation in Parkinson's disease. Brain 2019; 142: 3592-3604

[83] Galbiati A, Verga L, Giora E et al. The risk of neurodegeneration in REM sleep behavior disorder: a systematic review and meta-analysis of longitudinal studies. Sleep Med Rev 2019; 43: 37-46

[84] Zhang X, Sun X, Wang J et al. Prevalence of rapid eye movement sleep behavior disorder (RBD) in Parkinson's disease: a meta and meta-regression analysis. Neurol Sci 2017; 38: 163-170

[85] Postuma RB, Iranzo A, Hu M et al. Risk and predictors of dementia and parkinsonism in idiopathic REM sleep behaviour disorder: A multicentre study. Brain 2019; 142: 744-759

[86] Noyce AJ, R'Bibo L, Peress L et al. PREDICT-PD: an online approach to prospectively identify risk indicators of Parkinson's disease. Mov Disord 2017; 32: 219-226

[87] Berg D, Postuma RB, Adler CH et al. MDS research criteria for prodromal Parkinson's disease. Mov Disord 2015; 30: 1600-1611

[88] Heinzel S, Berg D, Gasser TM et al. Prodromal PD calculator (Members only). Im Internet: www.movementdisorders.org/pdcalculator. Stand: 29/01/2020

[89] Postuma RB, Berg D. Prodromal Parkinson's disease: the decade past, the decade to come. Mov Disord 2019; 34: 665-675

[90] Blumenberg C, Barros AJD. Electronic data collection in epidemiological research: The use of REDCap in the pelotas birth cohorts. Appl Clin Inform 2016; 7: 672-681

[91] Califf RM. Biomarker definitions and their applications. Exp Biol Med 2018; 243: 213-221

[92] Parnetti L, Gaetani L, Eusebi P et al. CSF and blood biomarkers for Parkinson's disease. Lancet Neurol 2019; 18: 573-586

[93] Redenšek S, Dolžan V, Kunej T. From genomics to omics landscapes of Parkinson's disease: revealing the molecular mechanisms. Omi A J Integr Biol 2018; 22: 1-16 